Archives

17| 1996

Hommage à Bernard Lepetit

\title{
Les ingénieurs militaires et l'aménagement du territoire. Le cas du réseau des places fortes $d u$ nord de la France aux XVII ${ }^{\mathrm{e}}$ et XVIII ${ }^{\mathrm{e}}$ siècles
}

Jean-Michel Manivit

\section{OpenEdition}

\section{Journals}

Édition électronique

URL : http://journals.openedition.org/ccrh/2592

DOI : $10.4000 /$ ccrh.2592

ISSN : 1760-7906

Éditeur

Centre de recherches historiques - EHESS

Édition imprimée

Date de publication : 4 octobre 1996

ISSN : 0990-9141

Référence électronique

Jean-Michel Manivit, « Les ingénieurs militaires et l'aménagement du territoire. Le cas du réseau des places fortes du nord de la France aux XVII et XVIII siècles ", Les Cahiers du Centre de Recherches Historiques [En ligne], 17| 1996, mis en ligne le 27 février 2009, consulté le 01 mai 2019. URL : http:// journals.openedition.org/ccrh/2592 ; DOI : 10.4000/ccrh.2592

Ce document a été généré automatiquement le 1 mai 2019.

Article L.111-1 du Code de la propriété intellectuelle. 


\title{
Les ingénieurs militaires et l'aménagement du territoire. Le cas du réseau des places fortes du nord de la France aux XVII et XVIII siècles
}

\author{
Jean-Michel Manivit
}

Ma rencontre avec Bernard Lepetit, avant nos contacts réguliers, évoque des moments décisifs bien qu'espacés. Au début des années 1980, face à lui, dans une reprise d'exposé d'agrégation, je me souviens de sa voix agréable distillant l'art de vous rendre intelligent en vous signifiant si gentiment vos lacunes. Cette pédagogie me devint si essentielle qu'elle influença à jamais mon enseignement dans le secondaire. Six ans plus tard, tandis que je le retrouvais, par hasard, à l'EHESS, avec l'impression de l'avoir quitté la veille, je goûtais avec plaisir ce sentiment de complicité dans l'ironie, mélange subtil de proximité et de distance qui marqua par la suite nos rapports.

Dès lors il ne manqua jamais ni de m'orienter dans mes recherches en urbanisme ni de m'informer. Des économistes m'avaient fait espérer un financement pour une thèse sur l'impact économique des places fortes sur l'aménagement du territoire français. Ce sujet fut jugé trop historique. Je retrouvai alors Bernard Lepetit. Ce n'est que plus tard, lorsque je découvris, en séminaire, l'intérêt qu'il portait aux militaires dans ses recherches sur l'expédition d'Egypte, que j'osai lui soumettre mon sujet. Il tenait le travail d'Hélène Vérin sur les ingénieurs pour profondément novateur ${ }^{1}$, aussi me proposa-t-il d'articuler mon projet autour de la figure de l'ingénieur militaire dans son rapport à l'aménagement du territoire. 


\section{Démarches et limites}

1 L'hypothèse que les militaires ont, par profession, une pensée de l'aménagement du territoire lui paraissait évidente. Les démarches d'entrée dans le sujet étaient cependant multiples. Nous en sélectionnâmes trois. La première était susceptible de prolonger en aval celle mise en œuvre par $\mathrm{H}$. Vérin sur le mode de raisonnement et les conditions de la pratique cognitive des ingénieurs. La deuxième consistait à suivre, à l'instar des travaux de A. Guillerme ${ }^{2}$, la manière dont les ingénieurs pensent, de façon systématique, les rapports entre les éléments, à l'échelle locale, autrement dit, le réseau local. Enfin la troisième s'attachait à scruter le discours des ingénieurs sur les places fortes et à confronter leurs réflexions théoriques aux rapports d'inspection enregistrés sur le terrain. L'écueil essentiel résidait dans une étude exhaustive de chacune des places fortes, au vu de l'immense fonds de l'article 8 des archives du génie, composé de plus de deux mille cartons, dans lequel s'était déjà noyé un thésard.

2 À la diversité des démarches correspond celle des limites chronologiques. En amont, Vauban est le point de départ de toute pensée sur l'espace, pour les ingénieurs militaires: il théorise le système des places fortes en réseau hiérarchisé et devient, tout au long des $\mathrm{XVIII}^{\mathrm{e}}$ et XIX ${ }^{\mathrm{e}}$ siècles, la référence obligée pour tous ses successeurs. La lettre de Vauban au roi, en 1672, indiquant la nécessité de transformer le nord de la France en un "pré carré ", représente non seulement une référence commode mais aussi une date fondatrice dans le système défensif français ${ }^{3}$. Les problèmes qu'il va rencontrer avec les élites locales n'empêcheront pas le XvIII ${ }^{e}$ siècle de réaliser en grande partie ses projets. En aval, les limites sont plus difficiles à tracer; en effet, si l'on s'en tient aux mémoires généraux, on constate que ni les débats animés par Guibert et d'Arçon durant la décennie 1780, au sujet de l'entretien des places fortes, ni les offensives révolutionnaires puis napoléoniennes ne rendent caduc l'intérêt porté aux places fortes et aux théories défensives. Cette tendance persiste, de l'occupation de Paris en 1815 à 1870 au moins, en passant par les débats qui précèdent l'édification de l'enceinte fortifiée parisienne de 1840.

3 L'unanimité en faveur de la place forte s'explique mieux lorsqu'on analyse, à travers la tourmente révolutionnaire et les conquêtes napoléoniennes, la continuité du corps des ingénieurs militaires. A. Blanchard a montré, à ce sujet, non seulement qu'une partie d'entre eux était restée aux commandes, mais qu'un petit noyau avait même tenté l'aventure politique à l'exemple de L. Carnot ou encore de Prieur de la Marne ${ }^{4}$.

4 La problématique générale des discours théoriques sur la place forte n'est pas suffisante pour borner strictement la chronologie du sujet. Aussi faut-il se recentrer sur des documents plus techniques, tels que les rapports d'enquête des ingénieurs, les projets d'amélioration des communications, sur le terrain, pour appréhender la science de l'ingénieur appliquée au territoire, notamment à travers son utilisation combinée des différentes échelles de la cartographie régionale ou locale.

5 La question du bornage chronologique rejoint alors celle de la délimitation de l'espace : le choix de la région du Nord s'impose car elle est la seule à faire l'objet d'un aménagement en réseau du fait de cette absence d'obstacles du relief qui autorise de fréquentes invasions, depuis le $\mathrm{XVI}^{\mathrm{e}}$ siècle. Ce sont donc les projets d'aménagement des voies de communication et leurs réalisations qui limiteront la chronologie de notre travail. Or, 
l'importance et la fréquence de ceux-ci dans les archives s'estompent à la fin du XVIII siècle, avec un regain très provisoire durant la période napoléonienne.

\section{La définition du réseau des places fortes}

6 Préciser le sens de quelques termes omniprésents, et tout particulièrement de la notion de réseau, s'avère indispensable, tant celle-ci a des accents qui fleurent l'anachronisme. Et pourtant, les définitions des géographes du groupe RECLUS (qui considèrent le réseau comme l'association articulée du point, de la ligne et de la surface) s'appliquent assez bien aux traités théoriques sur les places fortes de Vauban, Cormontaigne ou Fourcroy. Ceux-ci définissent les places fortes dans l'espace comme des points fortifiés, les uns par rapport aux autres, capables de se défendre mutuellement, en fonction de la portée de leurs canons respectifs, d'intervalles idéalement calculés (6 ou 8 lieues) et de positions fixées en quinconce sur plusieurs lignes de profondeur.

7 Ces points sont reliés entre eux par des lignes qui sont autant de voies de communication dont le schéma général, défini par Vauban, sera réalisé au cours du siècle suivant. Il s'agit, dans ce cas, de redessiner les axes majeurs de circulation de la région Nord, en infléchissant la direction des flux économiques. Traditionnellement orientés le long des fleuves rejoignant la mer du Nord (c'est-à-dire en direction des Pays-Bas, aux mains de l'ennemi espagnol), ces flux doivent suivre, désormais, une direction est-ouest qui a pour intérêt essentiel de relier entre elles les places fortes de première ligne, de Valenciennes à Dunkerque. Dans ce but, Vauban tente d'imposer un mode de communication jugé, tout à la fois, plus rapide et plus économique : la voie fluviale et/ou canalisée.

Enfin, l'espace de ce réseau se conçoit à l'échelle locale, à travers l'aire de rayonnement de chaque place forte, définie par sa zone d'inondation. Celle-ci constitue son périmètre de protection et délimite aussi bien le tracé de la frontière proche que le cadre légal de l'autorité d'action des ingénieurs militaires (surtout quand ceux-ci viendront à être concurrencés, dans leur maîtrise du territoire, par le corps des Ponts et Chaussées).

Qui plus est, ce réseau ne prétend pas être fixé de manière définitive, avec un point central à la tête d'une hiérarchie pyramidale, si cher aux théories du point fixe, en vigueur chez les philosophes du XVII ${ }^{\mathrm{e}}$ siècle, mais comme un système réversible et non plus statique. La place forte est alors conçue pour être un point successivement défensif et offensif, délimitant à la fois fortement l'espace par sa visibilité, mais aussi déterminant, pour ses alentours soumis à ses contre-attaques, un territoire extensible. Sa position topographique n'en fait plus un point superbement isolé sur un promontoire mais un carrefour situé dans une zone submersible ou marécageuse. Sa vocation n'est même pas d'être imprenable puisqu'elle est programmée pour résister un temps donné en immobilisant un ennemi qui peut lui être dix fois supérieur. Enfin leur nombre est susceptible de varier en fonction des traités et des remodelages de la frontière, ou bien même de l'évolution des places fortes ennemies.

10 L'existence de chaque place forte dans le réseau est, de plus, soumise à des impératifs de rendement: combien fait-elle économiser d'hommes de troupe, de places fortes à l'intérieur du pays ; combien fait-elle vivre de civils ?

11 Au total, il faut se débarrasser de l'idée d'associer les places fortes à la notion de territoire sanctuarisé et de défense linéaire, comme on est tenté de le faire, à partir de l'exemple de la ligne Maginot, dernier exemple de système fortifié; celui-ci apparaît au regard du 
système vaubanien comme une formidable régression dans la capacité de penser un espace. Il faut tout autant se méfier de la notion de hiérarchie, au sujet du classement des places fortes en premier, deuxième ou troisième rang, qui fait pourtant l'objet, chez les théoriciens militaires, de constantes discussions. Certains militaires, tel le général Haxo, rappellent à l'envi, en 1817, que la manie du classement ne résiste pas aux contingences de la guerre, à l'exemple de Landrecies, petite place forte, arrêtant en 1709 le prince Eugène, quand toutes les principales places fortes ont capitulé.

Un paradoxe vient cependant troubler cette vision moderne du réseau : bien que conçue pour se projeter vers l'extérieur de son territoire, la place forte ne peut s'étendre ni se développer à l'extérieur de ses propres murailles.

\section{Cartographie et jeux d'échelle} pistes de recherche s'imposa. Bernard Lepetit me poussa à explorer plus systématiquement la notion d'échelle, indissociable de la cartographie. cartographique, il sembla nécessaire de l'associer avec celles déjà abordées de point, ligne, surface. Précisons d'emblée (surtout au vu des archives du génie) que le domaine d'action des ingénieurs militaires a de plus en plus tendance à se confondre avec la maîtrise hydraulique du territoire.

En premier lieu, les ingénieurs militaires proposent des projets de régularisation ou de canalisation des rivières, telles la Scarpe ou la Deule. L'enjeu cartographique est avant tout linéaire, mais les incidences débordent toujours les aspects militaires pour atteindre les préoccupations économiques. Ainsi l'aménagement de ces rivières doit, à la fois, permettre de fournir un débit suffisant à la navigation qui approvisionne les places fortes ou les forts situés sur son passage, et procurer suffisamment d'eau aux zones inondables, à proximité. On doit prévoir dans le même temps les points où l'inondation pourrait être saignée par le drainage effectué par l'ennemi ainsi que des réservoirs destinés à l'inondation entre les places fortes de Lille et de Douai.

Dans d'autres cas, les motivations sont prioritairement hygiéniques, comme à l'occasion du détournement des eaux infectées des rouissoirs de l'abbaye et du village de SaintAmand-les-Eaux ou bien lors de l'assèchement des marais de la grande moere. De manière plus inédite, les canaux sont aussi envisagés pour constituer des lignes de défense fortifiées, ou protégées par les murs, à l'exemple du canal de Neuffossé entre Saint-Omer et Gravelines, dans le projet de 1751.

L'aménagement ponctuel reste généralement réservé à la place forte et à son environnement immédiat, et rentre alors dans le cadre de la limitation des interventions imposée aux ingénieurs militaires depuis que le corps des ingénieurs civils des Ponts et Chaussées s'est vu attribuer la maîtrise de l'aménagement routier. Ainsi, le port de Dunkerque, pourtant désarmé après le traité de 1713, fit l'objet de multiples projets d'aménagement d'écluses, batardeaux et canaux, tandis qu'à Lille on chercha à courtcircuiter la rupture de charge de la Deule, en centre-ville, par un canal de contournement longeant la citadelle.

18 Cependant les cartes des ingénieurs militaires peuvent dépasser largement leurs attributions et concerner une région entière où la complexité des phénomènes 
hydrauliques, comme dans la basse plaine de Flandre, leur permet une maitrise totale des communications dont l'unité de base est le watergang. Ils peuvent dresser aussi, de Dunkerque à Valenciennes, une carte linéaire du système des canaux perpendiculaires aux grands axes fluviaux.

Dans presque tous les cas, la cartographie est au centre d'une technologie territoriale minutieuse où s'imposent, avant tout, les lieux de communication et d'échange, les coupes et profils des rivières, sas et ponts, la praticabilité des marais, mais où disparaitt la perspective cavalière qui représentait auparavant les villages.

À ce stade, il serait intéressant d'établir une comparaison avec les cartes levées par les ingénieurs civils, en s'attachant pour chacune au choix des figurés, légendes, échelles et au style, ainsi qu'à la précision et à l'allure de scientificité. Il faudrait, pour ce faire, comparer des cartes d'une même aire géographique pour vérifier si l'aménagement hydraulique n'est pas devenu un terrain d'action où les ingénieurs militaires se replient. En effet, dans la région du Nord, très peu de projets routiers font l'objet d'une représentation cartographique à l'exception notable de la forêt de Mormal.

Les représentations de la frontière doivent aussi faire l'objet d'une étude et sa place dans l'aménagement de la région du Nord doit être envisagée en rapport avec les places fortes ennemies car, au cours du "paisible " XVIII ${ }^{\mathrm{e}}$ siècle, elles n'ont pas manqué de changer plusieurs fois de maître. C'est alors qu'il faudrait envisager l'étude de l'unité d'un nouveau type d'espace : le territoire frontalier.

\section{La science pragmatique de l'ingénieur}

L'approche qui m'a cependant le plus occupé lors de mes dépouillements concerne les modalités de la mise en pratique des conditions du savoir chez l'ingénieur militaire. Vauban, par ses inlassables visites sur le terrain préalablement cartographié, a contribué lui-même à définir la science appliquée de l'ingénieur dont il fut l'infatigable et intraitable pédagogue. Au-delà du va-et-vient constant entre un savoir théorique à base de mathématiques, acquis à partir de 1748 à l'école du génie de Mézières, et une pratique de terrain, c'est la constante nécessité de concilier des paramètres contradictoires qui me semble caractériser la science de l'ingénieur militaire. Celui-ci communique avec des catégories d'acteurs aux intérêts divergents ou à la logique conquérante.

Dans la première catégorie, il faut placer les élites locales, qui, dans l'exemple du cas lillois, luttent contre la tentative de contournement de la ville au nom de la défense des métiers menacés de disparition (portefaix); ou bien encore, les états d'Artois qui protestent quand les ingénieurs bloquent le projet routier de Lillers à Saint-Venant. Il faut dire que les solutions de Vauban pour Lille s'accompagnaient d'un financement inadapté : en refusant le péage, il voulait faire payer le voisinage, qui n'en profitait généralement pas.

Dans la seconde catégorie, on retrouve le corps des Ponts et Chaussées, allié aux états pour rappeler expressément les limites du champ d'action des militaires. Cela tend à infirmer l'idée d'un partage réel des compétences entre les catégories d'ingénieurs. On observe plutôt des tentatives d'empiétement, qui s'expliquent d'autant mieux que les ingénieurs militaires se retrouvent au $\mathrm{XVIII}^{\mathrm{e}}$ siècle dans un état de sous-activité et cherchent des fonctions d'expertise auprès des collectivités territoriales, qui font parfois appel à eux. 

et les poussent à raisonner en termes d'arbitrage ou de rapports coûts/avantages. Des choix doivent être faits entre la hausse du niveau de l'eau ou la baisse de la charge des bateaux, entre le creusement des canaux et la surélévation des digues. Le plus souvent, l'ingénieur doit concilier les impératifs contradictoires de la défense et de l'échange économique. Ainsi la nécessité, en 1807, de prélever de l'eau pour constituer des réservoirs d'inondation sur la haute Deule, entre Lille et Douai, risque de rendre le débit insuffisant, en été, pour la navigation et surtout de nuire aux usines et moulins situés dans la place forte de Douai.

Ailleurs, dans la forêt de Mormal - résidu des forêts-frontières du Moyen Âge devenues obstacles aux communications et, dans le cas présent, à l'exploitation du bois -, c'est un projet de percement de routes, par le corps des Ponts et Chaussées, en 1781, qui est jugé dangereux car il risque de faciliter la pénétration ennemie. Les ingénieurs militaires se doivent cependant de répondre par un contre-projet s'ils ne veulent pas passer pour des empêcheurs d'échanger et de prospérer en grand. Aussi c'est une solution encore une fois emblématique des modalités d'action du corps des ingénieurs militaires qui est avancée : la construction d'un canal perpendiculaire aux routes pénétrantes traversant la forêt entre la Sambre et l'Escaut contenterait le commerce et les militaires.

Au-delà de ces exercices de haute voltige, les ingénieurs militaires pensent une autre dimension de l'aménagement du territoire : celle des normes qu'ils tentent d'imposer aux bateaux susceptibles de circuler sur l'ensemble des canaux du Nord. Deux aspects méritent l'examen de ce projet qui mobilise plusieurs mémoires de 1722 à 1748 .

Tout d'abord, on retrouve, à travers le rêve d'uniformisation du réseau, l'ensemble des démarches colbertiennes et absolutistes. D'une part, la définition d'un type de bateau inspiré du modèle hollandais présente la capacité de naviguer en Manche en évitant toute rupture de charge ; d'autre part, la localisation, en un lieu unique, à Condé - en aval du confluent de l'Escaut et de la Scarpe, c'est-à-dire au croisement des axes sud-nord et ouest-est-, d'une manufacture de production, permet à la fois de mieux contrôler les normes de fabrication et d'offrir un site stratégique à proximité de la matière première de la forêt des Ardennes. Enfin, la distribution de privilèges pour les bateliers, accompagnée d'un système d'amendes pour tout retard d'acheminement, doit rendre le projet viable et rentable. Ce rêve de contrôle total aurait de quoi rendre ombrageux plus d'un technocrate d'aujourd'hui.

29 Secondairement, cette volonté de réduire les usages des pays aux normes royales s'ordonne autour de méthodes peu spectaculaires mais efficaces, faites de calculs pour trouver le meilleur ratio taille/volume, encombrement/tirant d'eau, vitesse/maniabilité, conjugués avec une savante prospective pour calculer le temps nécessaire à l'introduction de ces nouveaux bateaux et au renouvellement total de la flotte, temps estimé à vingt ans.

30 À la fin de cette réflexion, quelques pistes de travail fournissent des ouvertures intéressantes, mais des difficultés apparaissent aussi. Il faut créer une grille de lecture qui permette de faire entrer, dans un même système explicatif, les ensembles cartographiques et les multiples rapports et mémoires. Devant l'exposé de mes doutes quant à la possibilité de constituer des séries scientifiquement significatives, Bernard Lepetit, lors de notre dernière rencontre, me répondit avec une tranquillité provocante que le puzzle se constituerait finalement de lui-même, et j'avais une telle confiance dans ce qu'il pouvait tirer de ma pensée vagabonde, que j'étais prêt à le croire. 


\section{NOTES}

1. L'ingénieur est devenu une figure exemplaire de la pratique de l'histoire pragmatique telle que la revendiquait Bernard Lepetit dans le «tournant critique » de 1989. Pour une synthèse sur la genèse de ce mouvement, $\mathrm{Ch}$. Delacroix, in Espaces Temps, $\mathrm{n}^{\circ}$ 59-60-61.

2. Le Temps de l'eau, la cité, l'eau et les techniques, Seyssel, Champ Vallon, coll. «Milieux », 1990, $263 \mathrm{p}$.

3. Joël Cornette, Le Roi de guerre, Payot, 1994, montre combien le modèle hollandais en matière économique et de défense avait influencé Louis XIV. La fin de la guerre avec les Provinces-Unies, en 1672, marque une rupture dans la représentation du pouvoir.

4. A. Blanchard, "Les ci-devant ingénieurs du roi, essai de sociologie historique ", Revue internationale d'histoire militaire, $\mathrm{n}^{\circ}$ 30, $\mathrm{p}$ 97-107, 1970.

\section{AUTEUR}

\section{JEAN-MICHEL MANIVIT}

Maître en histoire contemporaine, université de Paris I, 1983. DEA « Histoire et civilisations ", EHESS. DEA d'urbanisme, université de Paris XII, 1991.

EHESS, sous la direction de Bernard Lepetit : Doctorant.

Professeur d'histoire, lycée. 